\title{
Clinical and immunological characteristics in COVID-19 convalescent patients
}

\author{
Zhiying Su ${ }^{1} \cdot$ Wenjia Hu ${ }^{1}$ Q Qianyun Liu ${ }^{2} \cdot$ Yongxi Zhang ${ }^{1} \cdot$ Tielong Chen ${ }^{1} \cdot$ Yingying Zhou $^{1} \cdot$ Zhen Zhang $^{2} \cdot$ Yu Chen $^{2}$. \\ Rongrong Yang ${ }^{1} \cdot$ Yong Xiong ${ }^{1} \cdot$ Xinghuan Wang $^{3}$
}

Received: 27 July 2021 / Accepted: 1 October 2021 / Published online: 9 October 2021

(c) The Author(s), under exclusive licence to Springer-Verlag GmbH Germany, part of Springer Nature 2021

\begin{abstract}
The humoral and cellular immunity of convalescent COVID-19 patients is involved in pathogenesis and vaccine immunity. In this study, through $\mathrm{CoV}-\mathrm{psV}$ neutralization assay and IFN- $\gamma$ ELISpot testing in 30 cases of COVID-19 patients after 9 months post-SARS-CoV-2 infection, it found that the ratio of memory/naive $\mathrm{CD}^{+} \mathrm{T}$ lymphocytes cells and levels of anti-SARSCoV-2-IgM and RBD-IgM were slightly but significantly higher in COVID-19 severe convalescent patients than that in nonsevere patients. The specific cellular and humoral immunity against SARS-CoV-2 were detectable, regardless of the severity of the disease in the acute phase. This information may help understanding the immune status after SARS-CoV-2 infection.
\end{abstract}

Keywords SARS-CoV-2 $\cdot$ Virus-specific T cells $\cdot$ Neutralizing antibodies $\cdot$ Humoral immunity

\section{Introduction}

COVID-19 is currently a major public health issue of international concern. In the context of the widespread global vaccination of COVID-19 to establish herd immunity, recent studies have been focused on whether a single dose

Zhiying Su, Wenjia Hu and Qianyun Liu contributed equally to this study. Yu Chen, Rongrong Yang, Yong Xiong, and Xinghuan Wang are correspondence authors and they contributed equally to this study.
Yong Xiong
yongxiong64@163.com
Zhiying Su
zyingsu@163.com
Wenjia Hu
huwenjia0820@163.com
Qianyun Liu
liuqianyun@whu.edu.cn
Yongxi Zhang
znact1936@126.com
Tielong Chen
530038676@qq.com
Yingying Zhou
15797677950@163.com
Zhen Zhang
zhangzhen@whu.edu.cn

of COVID-19 vaccine can establish an adequate protective barrier for previously SARS-CoV-2 infected persons [1-5]. Therefore, it is of great significance to investigate the specific cellular immunity and humoral immunity during the convalescence period after SARS-CoV-2 infection.

Most severe COVID-19 patients developed lymphopenia as well as pneumonia with higher plasma levels of pro-inflammatory cytokines [6-8], suggesting that the host immune system is involved in pathogenesis. Studies reported by Zhou et al. [9] and Krammer et al. [10] found that sera

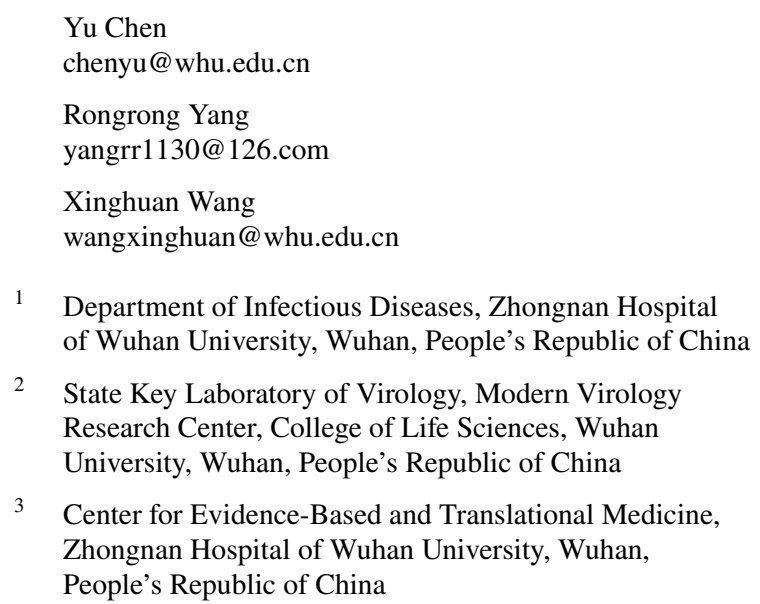

1 Department of Infectious Diseases, Zhongnan Hospital of Wuhan University, Wuhan, People's Republic of China

2 State Key Laboratory of Virology, Modern Virology Research Center, College of Life Sciences, Wuhan University, Wuhan, People's Republic of China

3 Center for Evidence-Based and Translational Medicine, Zhongnan Hospital of Wuhan University, Wuhan, People's Republic of China 
of COVID-19 patients could inhibit SARS-CoV-2 entry in target cells, indicating the involvement of humoral immunity. A study from Finland found a low level of neutralizing antibody titer in COVID-19 patient [11]. SARS-CoV2-specific antibody responses have been identified in several studies [12-14]. However, to what extent adaptive immune responses to SARS-CoV-2 infection associate with the clinical course of COVID-19 remains unclarified. How long the neutralizing antibodies can sustain after SARS-CoV-2 infection and their relationships with the severity of the disease also needs to be understood.

In this study, we followed up with COVID-19 patients who were discharged about 9 months post-SARS-CoV-2 infection. The relation of SARS-CoV-2-specific antibody and $\mathrm{T}$ cell responses with the severity of disease was reported.

\section{Methods}

\section{Study design and participants}

All patients with COVID-19 enrolled in this study were diagnosed according to World Health Organization interim guidance [15]. They were initially admitted to Zhongnan Hospital of Wuhan University from 21 January to 3 March 2020. In severity assessment on admission, severe COVID19 was defined according to a previous study [16]. They were treated at our hospital throughout the whole course of the disease and completed the follow-up test within the specified time. In another 16 cases, sex- and age-matched healthy blood donors were included as healthy control. The source of study subjects in this study was shown in Fig. 1.

\section{Flow cytometry and detection of SARS-CoV-2-specific IgG and IgM}

The $\mathrm{CD} 3^{+} / \mathrm{CD} 4^{+} / \mathrm{CD} 8^{+}$T-cell, $\mathrm{CD} 19^{+} \mathrm{B}$-cell, and $\mathrm{CD} 16^{+} \mathrm{CD} 56^{+} \mathrm{NK}$-cell counts testing procedure were performed according to a previously study [17]. The detection of SARS-CoV-2-specific IgG and IgM was performed according to the manufacturer's instructions. An antibody concentration of $\geq 10.0 \mathrm{AU} / \mathrm{mL}$ was considered reactive and of $<10.0 \mathrm{AU} / \mathrm{mL}$ non-reactive.

\section{Coronavirus spike protein pseudotyped virus (CoV-psV) neutralization assay}

Coronavirus spike protein pseudotyped virus (CoV-psV) were packaged following a previously described protocol using a replicate-deficient VSV-based rhabdoviral pseudotyping system (VSV-dG) [18]. Serial dilutions of nanobodies were mixed with pseudoviruses, incubated for $30 \mathrm{~min}$ at room temperature, and then added to Caco- 2 cells in a 96-well plate. Sixteen- to twenty-four hours later, cells were lysed by $1 \times$ passive lysis buffer (Promega, United States) at room temperature for $15 \mathrm{~min}$. Luciferase activity in the cell lysate was determined by a Bright-Glo luciferase assay kit (Promega, United States) and measured through a GloMax 20/20 Luminometer (Promega, United States).

\section{Interferon gamma (IFN- $\gamma$ ) ELISpot}

ELISpot assay procedures were referred to as described according to the previous report [19]. The spot increment $\geq 6$ was considered positive. However, when the numbers of
Fig. 1 Flowchart of the study subjects
507 COVID-19 patients hospitalized in Zhongnan Hospital of Wuhan University during 22 Jan and 3 Mar, 2020

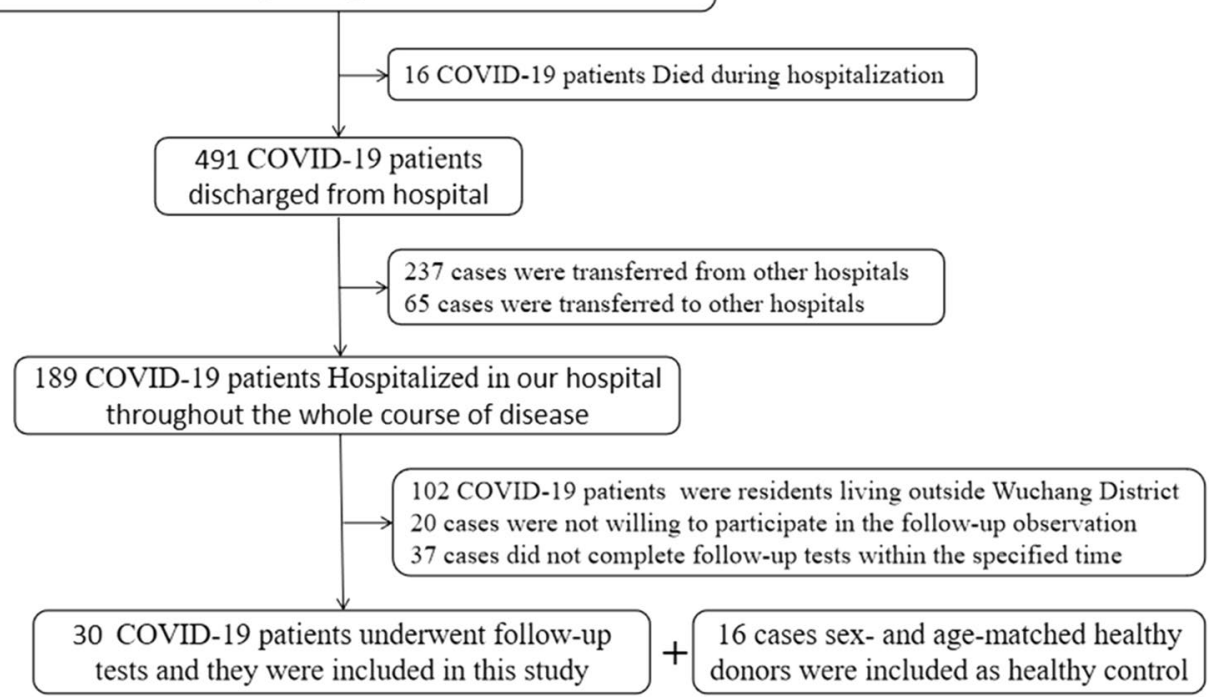


Fig. 2 Comparison of lymphocyte subtypes in the acute and convalescent phase
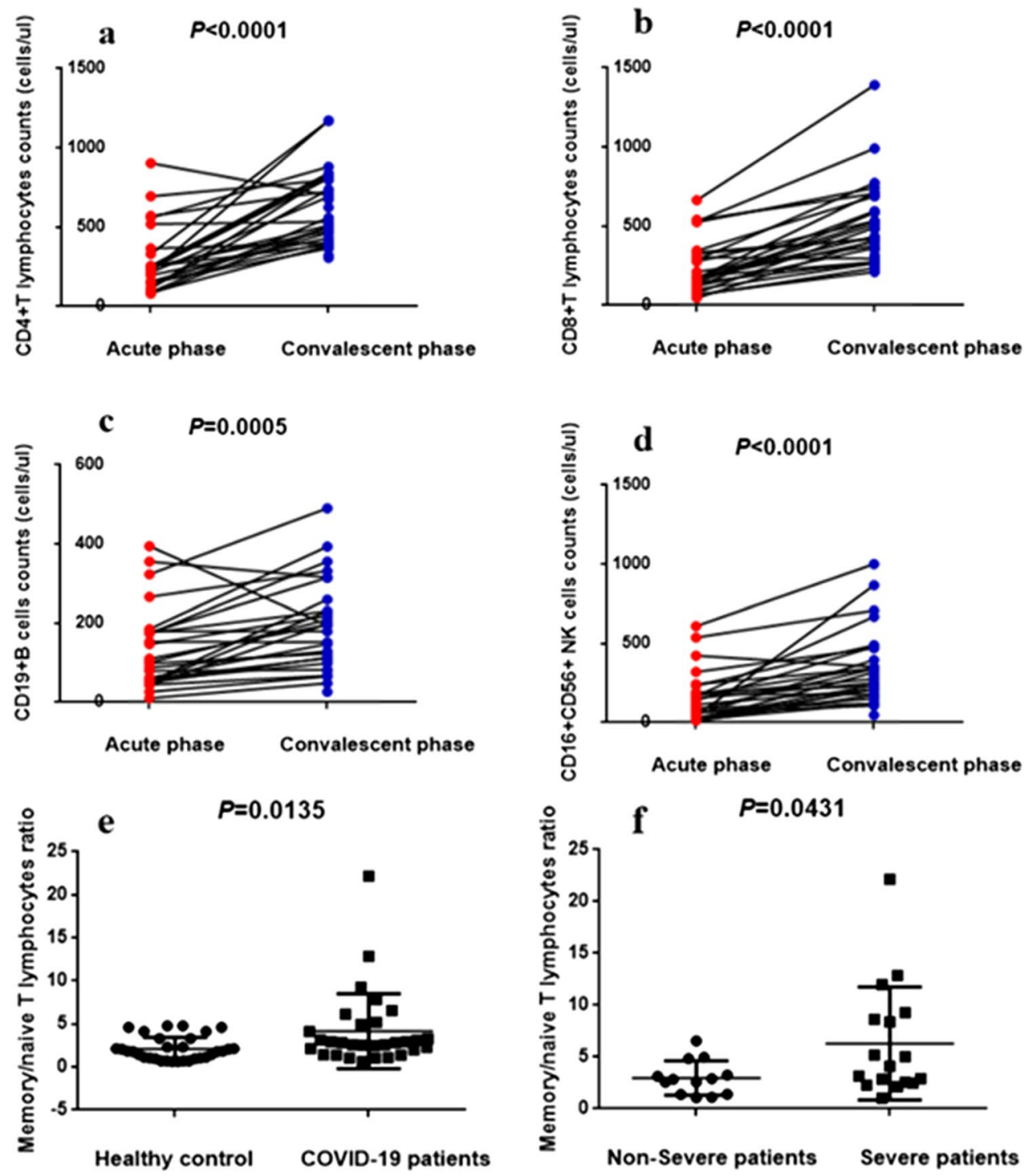

negative (unstimulated) spot forming units $(\mathrm{SFU}) \geq 6$, stimulated spot numbers $\geq$ twofold higher than negative (unstimulated) controls to any of the antigens were considered positive.

\section{Statistical analysis}

Categorical variables were described as frequency rates and percentages, and continuous variables were described using mean, median, and interquartile range (IQR) values. $\chi^{2}$ analysis or Fisher exact test was conducted to examine the categorical variables. Means for continuous variables in this study were compared using independent group $t$ tests when the data were normally distributed; otherwise, the Mann-Whitney test was used. Two-sided $p$ values $<0.05$ were considered statistically significant.

\section{Results}

\section{Patient characteristics}

Of the 30 COVID-19 convalescent patients, the median age was 56 \pm 12 years (range, 33-76 years) and 15 were male. Nine $(30.0 \%)$ had 1 or more comorbidity: hypertension (2 $(6.67 \%))$, diabetes $(1(3.33 \%))$, cardiovascular disease (2 $(6.67 \%))$, chronic kidney disease (1 $(3.33 \%))$, hypertension and diabetes coexisting conditions (2 (6.67\%)), and hypertension and cardiovascular disease coexisting conditions (2 $(6.67 \%))$. The age of the 16 healthy blood donors was comparable to those COVID-19 convalescent patients and 8 were male. Five (31.25\%) had comorbidity: hypertension (2 $(12.50 \%))$, diabetes (2 $(12.50 \%))$, and hyperthyroidism (1 $(6.25 \%))$. SARS-CoV-2-specific IgG and IgM were negative for all blood donors. 
Fig. 3 SARS-CoV-2-specific antibodies response in COVID19 convalescent patients by the severity of the disease. a The levels of anti-SARS-CoV-2$\operatorname{IgM}(P=0.014)$, anti-S1-IgM $(P=0.0004)$, and anti-RBD$\operatorname{IgM}(P=0.0002)$ in severe convalescent patients were higher than that in non-severe convalescent patients, except for the anti-N-IgM $(P=0.917) ; \mathbf{b}$ all the levels of $\mathrm{IgG}$ antibodies, including anti-SARS-CoV-2$\mathrm{IgG}$, anti-S1-IgG, anti-RBDIgG, and anti-N-IgG, were similar in convalescent patients, regardless of the disease severity in the acute phase a

Severe COVID-19 patients

ONon-Severe COVD-19 patients
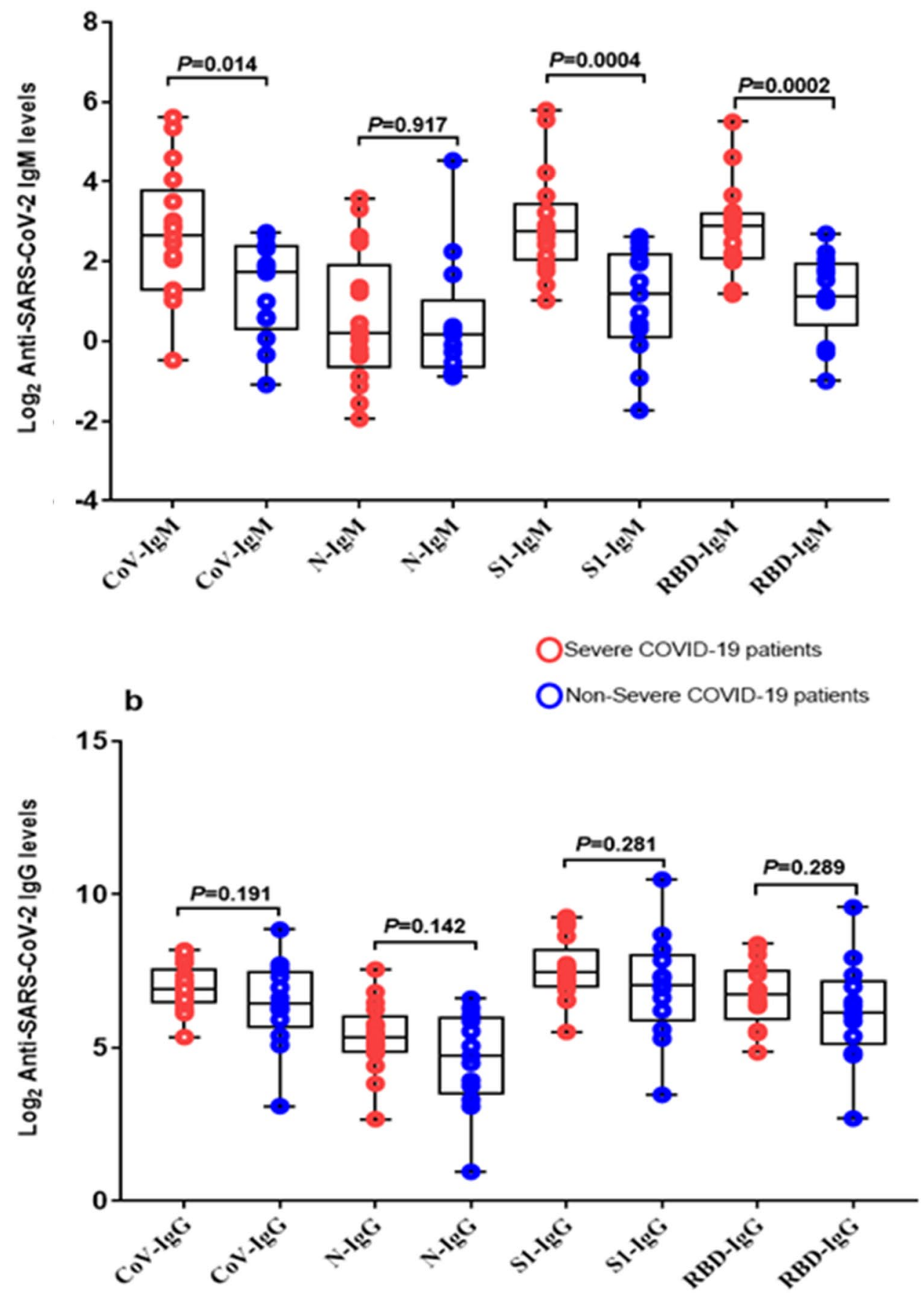

\section{Clinical laboratory findings}

A complete blood count, liver and renal function, inflammatory biomarkers, and coagulation function were measured in COVID-19 convalescent individuals to monitor the degree of recovery from SARS-CoV-2 infection. The results showed that the levels of white blood cells, lymphocyte counts, PLT,
BUN, ALT, Alb, GGT, LDH IL-6, CRP, PT, and D-Dimer were significantly improved and returned to normal levels in the convalescent phase. The clinical results of these above indicators were significantly different from those in the acute phase during hospitalization. 
Table 1 Follow-up results of anti-SARS-CoV-2 IgG and IgM between severe and non-severe patients at 283 days after the diagnosis of SARS-CoV-2 infection

\begin{tabular}{llllc}
\hline & Severe cases $(n=17)$ & $\begin{array}{l}\text { Non-severe cases } \\
(n=13)\end{array}$ & Test & $P$ value \\
\hline IgG & & & & \\
$\quad$ Total $\operatorname{IgG}(+)(n, \%)$ & $17(100 \%)$ & $12(92.3 \%)$ & 1.353 & 0.245 \\
N-IgG $(+)(n, \%)$ & $16(94.1 \%)$ & $10(76.9 \%)$ & 1.885 & 0.170 \\
S1-IgG(+) $(n, \%)$ & $17(100 \%)$ & $13(100 \%)$ & - & - \\
RBD-IgG $(+)(n, \%)$ & $17(100 \%)$ & $12(92.3 \%)$ & 1.353 & 0.245 \\
$\operatorname{IgM}$ & & & & \\
Total $\operatorname{IgM}(+)(n, \%)$ & $5(29.4 \%)$ & $0(0 \%)$ & 4.588 & 0.032 \\
$\mathrm{~N}-\operatorname{IgM}(+)(n, \%)$ & $1(5.9 \%)$ & $1(7.7 \%)$ & 0.039 & 0.844 \\
$\mathrm{~S} 1-\operatorname{IgM}(+)(n, \%)$ & $4(23.5 \%)$ & $0(0 \%)$ & 3.529 & 0.060 \\
$\mathrm{RBD}-\operatorname{IgM}(+)(n, \%)$ & $3(17.6 \%)$ & $0(0 \%)$ & 2.549 & 0.110 \\
\hline
\end{tabular}

\section{Lymphocyte subtypes}

Compared with the lymphocyte subtypes in the acute phase during hospitalization, the levels of $\mathrm{CD}^{+} \mathrm{T}$ lymphocyte cells (Fig. 2a), CD8 ${ }^{+}$T lymphocyte cells (Fig. 2b), CD19 ${ }^{+}$ B cells (Fig. 2c), and CD $16^{+} \mathrm{CD} 56^{+}$NK cells (Fig. 2d) significantly increased and returned to normal levels in convalescent phase, except for the ratio of memory/naive $\mathrm{CD} 4^{+} \mathrm{T}$ lymphocytes cells (Fig. 2e, $P=0.0135$ ). Moreover, the ratio of memory/naive CD4 ${ }^{+} \mathrm{T}$ lymphocytes cells in COVID-19 severe convalescent patients was higher than that in nonsevere patients (Fig. 2f, $P=0.0431$ ).

\section{SARS-CoV-2-specific antibodies}

The levels of anti-SARS-CoV-2-IgG, anti-SARS-CoV-2-NIgG, S1-IgG, and RBD-IgG in severe COVID-19 patients were similar to those in non-severe COVID-19 patients (Fig. 3a). Interestingly, except for N-IgM, the levels of
anti-SARS-CoV-2-IgM, S1-IgM, and RBD-IgM in severe COVID-19 patients were significantly greater than those in non-severe COVID-19 patients (Fig. 3b).

Moreover, all the 13 non-severe patients were negative for anti-SARS-CoV-2-IgM, whereas $29.4 \%$ (5/17) of the severe patients were positive for anti-SARS-CoV-2-IgM $\left(\chi^{2}=4.588, P=0.032\right)$ (Table 1).

\section{Serum neutralization capabilities in COVID-19 convalescent patients}

When the serums of COVID-19 convalescent patients were diluted as 1:125, the inhibition rate against SARS-CoV-2 infection was as high as $60 \%$, whereas the inhibition rate was less than $30 \%$ when the serums of healthy blood donors were diluted as 1:5. However, there was no significant difference in terms of the inhibition rate against SARS-CoV-2 infection among severe and non-severe COVID-19 patients (Fig. 4).
Fig. 4 Serum neutralization capabilities in COVID-19 convalescent patients (The inhibition rate against SARSCoV-2 infection in COVID-19 convalescent patients was as high as $60 \%$ even when their serums were diluted as 1:125, whereas an inhibition rate of less than 30\% was detected in healthy blood donors when their serums were diluted as 1:5.)

\section{VSVdG-SARS-2-S in Caco-2}

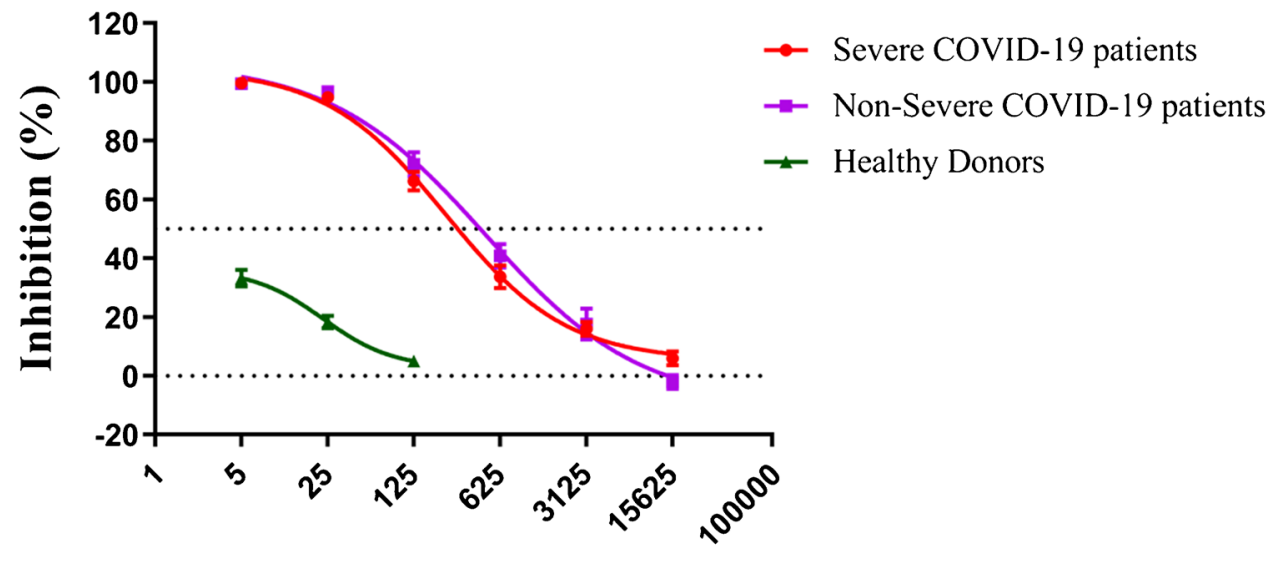

dilution multiple of serum 


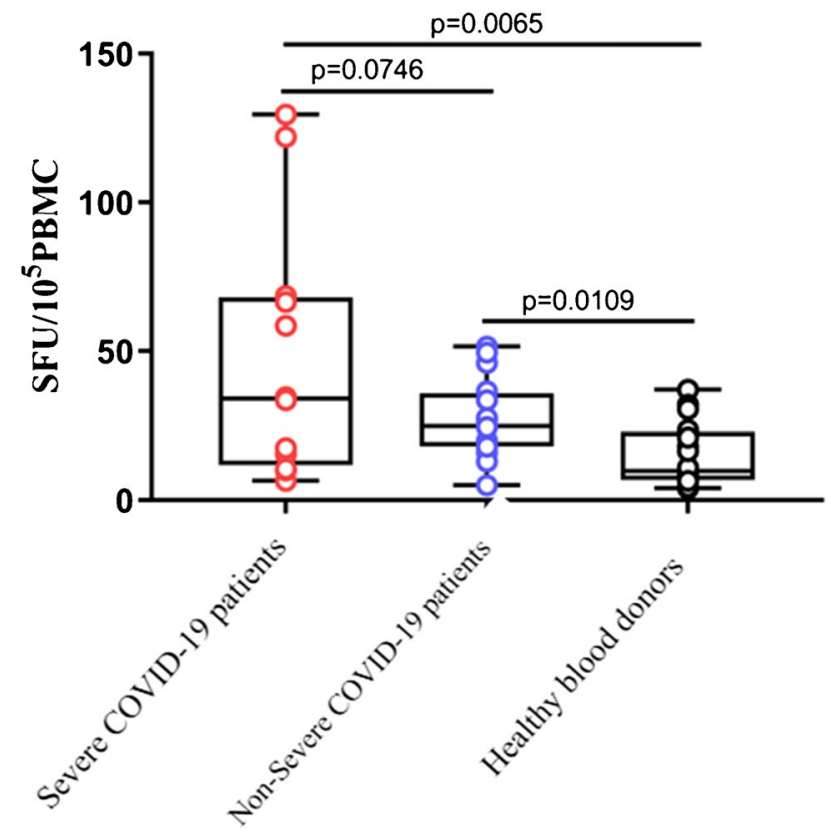

Fig. 5 SARS-CoV-2-specific $\mathrm{T}$ cellular immunity when stimulated by nucleocapsid protein(NP) in convalescent COVID-19 patients in relation to disease severity (Compared to healthy blood donors, more spot forming units by the IFN- $\gamma$ ELISpot test were found in convalescent COVID-19 patients. The spot forming units were similar in convalescent COVID-19 patients, regardless of the disease severity in the acute phase.)

\section{Proliferative capabilities of SARS-CoV-2-specific T cells in convalescent COVID-19}

In severe and non-severe COVID-19 convalescent patients, the proportion of SARS-CoV-2-specific T cells response was $82.4 \%$ (14/17) and 84.6\% (11/13), respectively. Moreover, the spot forming units by the IFN- $\gamma$ ELISpot test were $23 \pm 4$ and $30 \pm 7$ in the 14 severe and 11 non-severe COVID-19 convalescent patients, respectively. The spot forming units by the IFN- $\gamma$ ELISpot test was similar in severe and nonsevere COVID-19 convalescent patients (Fig. 5).

\section{Discussion}

In this follow-up study, as expected, we found complete blood cell count, liver and kidney function, coagulation function, and inflammatory cytokine levels of the patients all returned to normal levels. Interestingly, the ratio of memory/ naive $\mathrm{CD}^{+}{ }^{+} \mathrm{T}$ lymphocytes cells and levels of anti-SARSCoV-2-IgM and RBD-IgM in severe COVID-19 convalescent patients were slightly but significantly higher than that in non-severe cases. Importantly, it found that in the convalescence phase, SARS-CoV-2 infection patients had specific cellular immunity, and the specific antibodies had the capacity to neutralize SARS-CoV-2.

In this study, SARS-CoV-2 IgM was positive in $29.4 \%$ (5/17) severe COVID-19 convalescent individuals, whereas none was positive in non-severe patients. Further follow-up is needed to determine how long SARS-CoV-2 IgM lasts in severely COVID-19 patients, but a conclusion we can draw now was that the late disappearance of SARS-CoV-2 IgM may indicate a more serious condition.

As previously reported [8], SARS-CoV-2-specific humoral immunity was detected in newly discharged patients. In this study, we demonstrated that immunemediated protection to viral infection can be detected after 9 months post-SARS-CoV-2 infection. Recently, several studies characterizing adaptive immune responses to SARSCoV-2 infection have reported that most COVID-19 convalescent individuals have detectable neutralizing antibodies, which correlate with the numbers of virus-specific $\mathrm{T}$ cells $[8,9,20-23]$. This study further found that virus-stimulated neutralizing antibodies can sustain for several months, and the capacity to neutralize SARS-CoV-2 in severe convalescent patients was close to that in non-severe patients.

Antibody responses in patients previously infected with SARS-CoV tended to be short-lived [24], whereas SARS-CoV-specific memory $\mathrm{T}$ cells were found to persist for 6 years after infection in SARS survivors [25-27]. Memory $\mathrm{CD}^{+} \mathrm{T}$ cells have multiple roles in initiating and propagating the immune response [28]. SARS-CoV2 -specific $\mathrm{T}$ cells in the acute phase and the convalescent phase were proved to be different, displaying as a highly activated cytotoxic phenotype that correlated with various clinical markers of disease severity in the acute phase and a stem-like memory phenotype in the convalescent phase [29]. However, little is known about whether the proportion of these cells changed after SARS-CoV-2 infection. In this study, the ratio of memory/naive $\mathrm{CD} 4^{+} \mathrm{T}$ lymphocytes cells in severe COVID-19 convalescent patients was found to be greater than that in non-severe COVID-19 convalescent patients, which indicated that specific cellular immunity plays an important role in pathogenesis after SARS-CoV-2 infection. This potential effect can persist about 9 months post-SARS-CoV-2 infection. Whether this memory $\mathrm{T}$ response has a protective effect on SARS$\mathrm{CoV}-2$ re-infection is still worth further study.

We recognize that our study has limitations. Given the single-center study and the small number of samples, the representativeness of the outcome may be limited to some extent. Also, although we confirmed that humoral immunity responses and specific cellular immunity in COVID-19 convalescent patients can be detectable after 9 months of SARS-CoV-2 infection, whether the neutralizing antibodies and specific cellular immunity have a protective effect on 
Delta or Lambda variants is still worth exploring. Our team will try our best to make up for the defects in future studies.

\section{Conclusion}

The specific cellular and humoral immunity against SARSCoV-2 were detectable after 9 months post-SARS-CoV-2 infection, regardless of the severity of the disease in the acute phase. This information may help understanding the immune status after SARS-CoV-2 infection.

Author contribution Y.X. and X.W. conceptualized the study design; Z.S. and W.H. recruited the patients, collected specimens, collected demographic, and clinical data; Q.L., Z.S., Y.Z., Z.Z., and W.H. did the laboratory tests; T.C., R.Y., Y.Z., and Y.C. interpreted the results; R.Y. wrote the initial drafts of the manuscript; Y.C., R.Y., Y.X., and X.W. revised the manuscript. All authors read and approved the final manuscript.

Funding This work was supported by the Key Project for Anti-2019 Novel Coronavirus Pneumonia from the National Key Research and Development Program of China (Grant No. 2020YFC0845500 by Xinghuan Wang and 2018YFA0900801 by Yu Chen); COVID-19 Emergency Response Project of Hubei Province (Grant No. 2020FCA005 by Yong Xiong); Fundamental Research Funds for the Central Universities (Grant No. 2042020kf1018 and Grant No.TFJC2018002 by Yong Xiong); and National Natural Science Foundation of China (Grant No. 82003511 by Rongrong Yang and 32041007 by Yu Chen).

Data availability The datasets used or analyzed during the current study are available from the corresponding author on reasonable request.

Code availability Not applicable.

\section{Declarations}

Ethics approval This study was approved by the Ethics Committee of the Zhongnan Hospital of Wuhan University (No. 2020011).

Consent to participate Written informed consent to participate in this study can be obtained from all patients.

Consent for publication Written informed consent for publication can be obtained from all participants.

Competing interests The authors declare no competing interests.

\section{References}

1. Stamatatos L, Czartoski J, Wan YH et al (2021) mRNA vaccination boosts cross-variant neutralizing antibodies elicited by SARSCoV-2 infection. Science 372:1413-1418. https://doi.org/10.1126/ science.abg9175
2. Goel RR, Apostolidis SA, Painter MM et al (2021) Distinct antibody and memory B cell responses in SARS-CoV-2 naïve and recovered individuals following mRNA vaccination. Sci Immunol 6(58):eabi6950. https://doi.org/10.1126/sciimmunol.abi6950

3. Ebinger JE, Fert-Bober J, Printsev I et al (2021) Antibody responses to the BNT162b2 mRNA vaccine in individuals previously infected with SARS-CoV-2. Nature Med 27(6):981-984. https://doi.org/10.1038/s41591-021-01325-6

4. Krammer F, Srivastava K, Alshammary H et al (2021) Antibody Responses in Seropositive Persons after a Single Dose of SARSCoV-2 mRNA Vaccine. N Engl J Med 384(14):1372-1374. https://doi.org/10.1056/NEJMc2101667

5. Wang Z, Muecksch F, Schaefer-Babajew D et al (2021) Naturally enhanced neutralizing breadth against SARS-CoV-2 one year after infection. Nature 595(7867):426-431. https://doi.org/10.1038/ s41586-021-03696-9

6. Chan JF, Yuan S, Kok KH et al (2020) A familial cluster of pneumonia associated with the 2019 novel coronavirus indicating person-to-person transmission: a study of a family cluster. Lancet 395(10223):514-523. https://doi.org/10.1016/S0140-6736(20) 30154-9

7. Huang C, Wang Y, Li X et al (2020) Clinical features of patients infected with 2019 novel coronavirus in Wuhan. China Lancet 395(10223):497-506. https://doi.org/10.1016/S0140-6736(20) 30183-5

8. Wu F, Zhao S, Yu B et al (2020) A new coronavirus associated with human respiratory disease in China. Nature 579(7798):265269. https://doi.org/10.1038/s41586-020-2008-3

9. Zhou P, Yang XL, Wang XG et al (2020) A pneumonia outbreak associated with a new coronavirus of probable bat origin. Nature 579(7798):270-273. https://doi.org/10.1038/s41586-020-2012-7

10. Krammer F, Simon V (2020) Serology assays to manage COVID19. Science 368(6495):1060-1061. https://doi.org/10.1126/scien ce.abc1227

11. Haveri A, Smura T, Kuivanen S et al (2020) Serological and molecular findings during SARS-CoV-2 infection: the first case study in Finland, January to February 2020. Euro Surveill 25(11):2000266. https://doi.org/10.2807/1560-7917.ES.2020.25. 11.2000266

12. Grifoni A, Weiskopf D, Ramirez SI et al (2020) Targets of T cell responses to SARS-CoV-2 coronavirus in humans with COVID19 disease and unexposed individuals. Cell 181(7):1489-1501. https://doi.org/10.1016/j.cell.2020.05.015 (e15)

13. Ni L, Ye F, Cheng ML et al (2020) Detection of SARS-CoV2 -specific humoral and cellular immunity in COVID-19 convalescent individuals. Immunity 52(6):971-977. https://doi.org/10. 1016/j.immuni.2020.04.023 (e3)

14. Thevarajan I, Nguyen THO, Koutsakos M et al (2020) Breadth of concomitant immune responses prior to patient recovery: a case report of non-severe COVID-19. Nat Med 26(4):453-455. https:// doi.org/10.1038/s41591-020-0819-2

15. World Health Organization. Clinical management of severe acute respiratory infection when novel coronavirus $(\mathrm{nCoV})$ infection is suspected: interim guidance; 2020. https://www.who.int/publi cations-detail/clinical-managementof-severe-acute-respiratoryinfection-when-novelcoronavirus-(nCoV)-infection-is-suspected. Accessed January 312020

16. Yang R, Xiong Y, Ke H et al (2020) The role of methylprednisolone on preventing disease progression for hospitalized patients with severe COVID-19. Eur J Clin Invest 50(11):e13412. https:// doi.org/10.1111/eci.13412

17. Wang F, Nie J, Wang $\mathrm{H}$ et al (2020) Characteristics of peripheral lymphocyte subset alteration in COVID-19 pneumonia. J Infect Dis 221(11):1762-1769. https://doi.org/10.1093/infdis/jiaa150 
18. Heilingloh CS, Aufderhorst UW, Schipper L et al (2020) Susceptibility of SARS-CoV-2 to UV irradiation. Am J Infect Control 48(10):1273-1275. https://doi.org/10.1016/j.ajic.2020.07.031

19. Schwarzkopf S, Krawczyk A, Knop D et al (2021) Cellular immunity in COVID-19 convalescents with PCR-confirmed infection but with undetectable SARS-CoV-2-specific IgG. Emerging Infect Dis 27(1). https://doi.org/10.3201/2701.203772

20. Wu F, Liu M, Wang A et al (2020) Evaluating the association of clinical characteristics with neutralizing antibody levels in patients who have recovered from mild COVID-19 in Shanghai, China. JAMA Intern Med 180(10):1356-1362. https://doi.org/10. 1001/jamainternmed.2020.4616

21. Suthar MS, Zimmerman MG, Kauffman RC et al (2020) Rapid generation of neutralizing antibody responses in COVID-19 patients. Cell Rep Med 1(3):100040. https://doi.org/10.1016/j. xcrm.2020.100040

22. Long QX, Jia YJ, Wang X et al (2021) Immune memory in convalescent patients with asymptomatic or mild COVID-19. Cell Discov 7(1):18. https://doi.org/10.1038/s41421-021-00250-9

23. Wu J, Liang B, Chen $\mathrm{C}$ et al (2021) SARS-CoV-2 infection induces sustained humoral immune responses in convalescent patients following symptomatic COVID-19. Nat Commun 12(1):1813. https://doi.org/10.1038/s41467-021-22034-1

24. Channappanavar R, Zhao J, Perlman S (2014) T cell-mediated immune response to respiratory coronaviruses. Immunol Res 59(1-3):118-128. https://doi.org/10.1007/s12026-014-8534-z
25. Zhao J, Zhao J, Mangalam AK et al (2016) Airway memory CD4(+) T cells mediate protective immunity against emerging respiratory coronaviruses. Immunity 44(6):1379-1391. https:// doi.org/10.1016/j.immuni.2016.05.006

26. Le Bert N, Tan AT, Kunasegaran $\mathrm{K}$ et al (2020) SARS-CoV2-specific T cell immunity in cases of COVID-19 and SARS, and uninfected controls. Nature 584(7821):457-462. https://doi.org/ 10.1038/s41586-020-2550-Z

27. Tang F, Quan Y, Xin ZT et al (2011) Lack of peripheral memory B cell responses in recovered patients with severe acute respiratory syndrome: a six-year follow-up study. J Immunol 186(12):72647268. https://doi.org/10.4049/jimmunol.0903490

28. Swain SL, McKinstry KK, Strutt TM (2012) Expanding roles for $\mathrm{CD}^{+} \mathrm{T}$ cells in immunity to viruses. Nat Rev Immunol 12(2):136-148. https://doi.org/10.1038/nri3152

29. Sekine T, Perez-Potti A, Rivera-Ballesteros O et al (2020) Robust $\mathrm{T}$ cell immunity in convalescent individuals with asymptomatic or mild COVID-19. Cell 183(1):158-168. https://doi.org/10.1016/j. cell.2020.08.017

Publisher's note Springer Nature remains neutral with regard to jurisdictional claims in published maps and institutional affiliations. 